\title{
Local application of gentamicin collagen implants in the prophylaxis of surgical site infections following gastrointestinal surgery: a review of clinical experience
}

\author{
A. F. J. de Bruin • M. P. Gosselink • \\ E. van der Harst $\cdot$ H. J. T. Rutten
}

Received: 4 January 2010/Accepted: 10 June 2010/Published online: 29 June 2010

(C) The Author(s) 2010. This article is published with open access at Springerlink.com

\begin{abstract}
Background Surgical site infection (SSI) is a common type of healthcare-associated infection in gastrointestinal (GI) surgical procedures, which often has major consequences for patient recovery and increased healthcare costs due to prolonged hospital stay. This article provides an overview of the efficacy and safety of prophylactic application of resorbable gentamicin-containing collagen implants (GCI) in the prevention of SSI following high-risk GI surgical procedures.

Method Nine publications were identified using the PubMed online database and search terms 'gentamicin collagen implant' plus 'surgical site infection', 'wound infection' and 'gastrointestinal surgery'.

Results Data from 483 patients treated prophylactically have demonstrated that GCI can reduce the wound infection rate in high-risk GI surgical procedures and improve wound healing after pilonidal sinus excision. In a study of 221 patients who underwent colorectal surgery, the wound infection rate was reduced to $5.6 \%$ in the GCI group compared to $18.4 \%$ in the control group $(P<0.01)$. GCI also positively influences the post-operative course for patients undergoing particularly risky procedures e.g. abdominoperineal resection (APR) combined with neoadjuvant radiotherapy. In one such patient series, GCI
\end{abstract}

A. F. J. de Bruin $(\bowtie) \cdot$ M. P. Gosselink · E. van der Harst Department of Surgery, Division of Colon and Rectal Surgery, Medisch Centrum Rijnmond Zuid, Maasstad Ziekenhuis, Olympiaweg 350, 3078 HT Rotterdam, The Netherlands e-mail: anton_de_bruin@fssoftware.nl

H. J. T. Rutten

Department of Surgery, Catharina Hospital,

Eindhoven, The Netherlands reduced the wound infection rate by over $70 \%$ and the length of hospital stay by $40 \%$. Few side effects of GCI were noted in the 9 clinical studies.

Conclusions This review demonstrates that GCI can have a positive effect on wound infection rates in high-risk GI surgery and can also improve wound healing after pilonidal sinus excision.

Keywords Collagen - Collagen implant · Gentamicin . Rectal surgery - Surgical site infection · Wound infection

\section{Introduction}

Surgical site infection (SSI) is a common type of healthcare-associated infection, which often has major consequences for patient recovery and increased healthcare costs due to prolonged hospital stay.

The risk of a patient developing an SSI is related to comorbidities, type of procedure and other factors such as length of surgery and use of prosthetic implants [1-3]. Data from national and regional surveys and single studies have highlighted high SSI rates in gastrointestinal (GI) surgical procedures (e.g. elective colorectal resection) [4-6]. Some of the higher rates of SSI in GI surgery are associated with newer techniques aimed at improving local control and resectability of the tumour, e.g. pre-operative radiotherapy followed by abdominoperineal resection (APR) [7, 8]. Therefore, there is a need for better prevention of SSIs following GI surgery in order to improve patient morbidity and decrease healthcare costs.

The mainstay of SSI prevention in addition to strict aseptic surgical technique is the use of antibiotic prophylaxis administered systemically both pre- and postoperatively. 
Results from 4 individual studies contained within a systematic review showed consistently that the wound infection rate was much lower in the antibiotic groups than that in the control groups (13\% versus 40\%; OR 4.08, $95 \%$ CI (c.i) 2.33-7.13) [9]. However, following GI surgery there is a tendency to administer systemic antibiotics for longer time periods, despite the fact that this may not necessarily lower the risk of SSI and this may in turn lead to a greater risk of antibiotic resistance [9-12].

The emergence of technologies such as resorbable gentamicin-containing collagen implants (GCI), which deliver high local concentrations of gentamicin with correspondingly low serum levels offer a means of reducing SSI rates following GI surgery, lowering the risk of antibiotic resistance by reducing the need for long-term administration of systemic antibiotics, avoiding toxicity associated with systemic antibiotics, and avoiding the need for reoperation as the implant is fully resorbable. The use of collagen as a carrier also has positive effects on wound healing.

GCI seems to limit the risk of SSI in several ways. Collagen causes faster coagulation to stop bleeding [13] and reduces the risk of seroma and haematoma formation that can in turn accelerate bacterial proliferation [14, 15]. The breakdown of the GCI by macrophage collagenases increases the number of collagen fibres released, which attracts fibroblasts and stimulates the fibroblasts to proliferate and lay down new collagen in the healing process [13].

Immune responses against collagen implants are uncommon [13, 16, 17], whereas collagen implants act as effective drug carriers [18, 19]. Drugs are released from the collagen matrix by a combination of diffusion and natural enzymatic breakdown of the collagen matrix providing rapid (diffusion) and prolonged (breakdown of the matrix) drug release [19].

The antimicrobial profile of gentamicin seems to be appropriate for use with GCI. Gentamicin allows sterilisation of the implants by gamma rays and does not seem to affect the collagen clotting features, as discussed above $[16,20]$. The local use of gentamicin delivers a bactericidal concentration that is much higher than that allowed by systemic injection, so antibiotic blood concentrations remain low and reduce the chance of adverse effects (e.g. nephrotoxicity), while the local drug concentrations are kept high for at least $48 \mathrm{~h}[21,22]$. In this way, resistance to antibiotics caused by low drug dosage is avoided [23], and gentamicin behaves like a broad spectrum antibiotic [21, 23].

A number of resorbable GCI are available worldwide. The objective of this article is to review all published clinical data regarding prophylactic application of resorbable GCI following GI surgery in order to provide an overview of the efficacy and safety of GCI for specific indications with particular focus on high-risk procedures.

\section{Method}

The candidate publications were identified using the National Library of Medicine's PubMed online search utility and the search term 'gentamicin collagen implant' plus 'surgical site infection', 'wound infection' and 'gastrointestinal surgery'. This search identified 28 potential publications. Nine publications focusing specifically on GI surgery and related areas (e.g. pilonidal sinus resection) were selected. These studies concerned the use of two GCI i.e. Collatamp ${ }^{\circledR}$ (EUSA Pharma [Europe], Oxford, United Kingdom) and Septocoll ${ }^{\circledR}$ (Biomet Deutschland $\mathrm{GmbH}$, Berlin, Germany). Collatamp contains $2 \mathrm{mg} / \mathrm{cm}^{2}$ gentamicin sulphate. The collagen in Collatamp, present at $2.8 \mathrm{mg} /$ $\mathrm{cm}^{2}$, is type I collagen from a renatured bovine or equine source. Septocoll contains gentamicin sulphate $(70 \mathrm{mg}$ gentamicin from $116 \mathrm{mg}$ gentamicin sulphate base in a $10 \mathrm{~cm} \times 8 \mathrm{~cm}$ implant) and gentamicin crobefate $(70 \mathrm{mg}$ gentamicin from $350 \mathrm{mg}$ gentamicin crobefate in a $10 \mathrm{~cm} \times 8 \mathrm{~cm}$ implant). Septocoll contains type I collagen from a renatured equine source.

The outcome measures of primary interest were SSI rate (superficial and deep), primary and secondary healing rate together with post-operative and surgical complication rate. Other outcome measures include time to complete wound healing; operating time; length of hospital stay; tumour recurrence (local and distant); survival time; pain score; analgesic use and cost. The level of evidence for each study was graded according to the criteria developed by Carruthers et al. [24] (see Table 1).

\section{Clinical experience}

To date, there have been 9 clinical studies which have focused on the local application of GCI in the prophylaxis of SSI following gastrointestinal surgery (Table 2). Six studies were of what would be considered procedures at high-risk of SSI and three of procedures at medium-risk. Eight of the studies were of randomised, controlled design, and one included a consecutive patient series with contemporaneous controls. Seven of the studies were graded as level 1 (see Table 2). Taken together these studies represent experience with 483 patients treated prophylactically with GCI ( $n=383$ with Collatamp and $n=100$ with Septocoll) following GI surgery.

\section{Rectal surgery}

There is a growing body of data to support the use of GCI following high-risk GI surgical procedures to reduce wound infection rates, wound healing time and length of hospital stay. Three randomised trials have assessed the effect of GCI in rectal tumour resection. Rutten and 
Table 1 Levels of evidence for rating studies [25]

\begin{tabular}{ll}
\hline Level & Definition of evidence \\
\hline 1 & Randomised, controlled trial (RCT) that demonstrated a \\
statistically significant difference in at least one important \\
outcome \\
OR \\
If the difference is not statistically significant, an RCT of \\
adequate sample size to exclude a $25 \%$ difference in relative \\
risk with $80 \%$ power, given the observed results
\end{tabular}

colleagues undertook a randomised study in which 221 patients undergoing a range of colorectal surgical procedures were randomised to the application of GCI plus systemic antibiotics (Group I) or systemic antibiotics alone (Group II) (Table 2) [25]. There was a significantly lower rate of post-operative wound infection in Group I $(P<0.01)$. The difference between the 2 groups was maintained even when patients with multiple complications were excluded $(P<0.05)$. The patients in Group I also had a significantly shorter hospital stay when compared to those in Group II $(P=0.015)$. However, after exclusion of the patients with complications unrelated to wound infection, the difference between the two groups diminished, with the median stay in Group I being one day shorter than Group II (13 vs. 14 days, respectively).

A second randomised, single centre study, in a similar group of patients $(n=229)$ treated with application of GCI plus systemic antibiotics (Group I), or systemic antibiotics alone (Group II) [26] (Table 2), reported a significantly lower post-operative complication rate (including wound infection) at 30 days in Group I $(P=0.0441)$. Sub-group analysis also revealed that this difference was maintained in patients at even higher risk of post-operative complications i.e. in those whose surgery exceeded $3 \mathrm{~h}(P=0.0314)$ and those who experienced intraoperative bowel perforation $(P=0.01)$. The patients in Group I also had significantly lower rates of cancer recurrence (local or metastasic) $(P=0.03)$ and increased overall and disease-free survival (at 3 years). However, the reasons for this are unclear, and further studies are required to confirm these results and clarify the mechanisms involved.
A third randomised, single blind, multicentre study by Grüssner and colleagues focused specifically on 97 patients undergoing abdominoperineal resection (APR) of the anorectum, which is associated with a particularly high risk of infection and other post-operative complications as already discussed [27]. Patients were randomised to treatment with application of GCI; systemic antibiotics plus sacral drainage (Group I) or systemic antibiotics plus sacral drainage (Group II). The patients in Group I had a significantly lower rate of perineal wound infections $(P<0.05)$ and secondary complications with infection $(P<0.05)$ compared to those in Group II. In addition, patients in Group I had a much greater reduction in the number of pathogens in the post-operatively obtained wound secretion samples compared to Group II $(P=0.013)$.

In a recent publication, we studied for the first time the effect of GCI in patients undergoing APR following shortterm, neoadjuvant radiotherapy. This study consisted of a consecutive series of 40 subjects. All patients received pre-operative radiation ( 5 Gy daily for 5 days) followed by a typical APR using the total mesorectal excision technique. Patients were then treated with application of GCI, systemic antibiotics plus sacral drainage (Group I) or systemic antibiotics plus sacral drainage (Group II) [28] (Table 2). This study demonstrated significantly lower rates of wound infection $(P=0.05)$ and significantly higher rates of primary wound healing $(P<0.01)$ in Group I. The patients in Group I also had significantly shorter mean hospital stays compared to those in Group II $(P=0.04)$.

\section{Abdominal surgery}

Two randomised studies have assessed the effect of the prophylactic application of GCI in various types of abdominal surgery. Gomez and colleagues randomised 73 patients to the application of GCI plus systemic antibiotics (Group I) and primary wound closure or systemic antibiotics and an open wound technique for the prophylaxis of infection in abdominal wounds that were 'dirty'(e.g. due to colon perforation) (Group II) [29] (Table 2). Patients in Group I had a statistically significant lower wound infection rate $(P<0.001)$. The difference between the two groups showed that the application of GCI made prophylaxis four times more effective. Similarly, good results were also evident in Group I for time to wound healing; in Group I, patients took an average of 10.31 days to heal compared to 15.20 days in Group II $(P<0.001)$. Given the lower rate of wound infection and the quicker time to healing, patients in Group I spent an average of 1.55 days less in hospital compared to those in Group II.

A second, randomised study focused specifically on the effect of GCI on the incidence of wound infection 


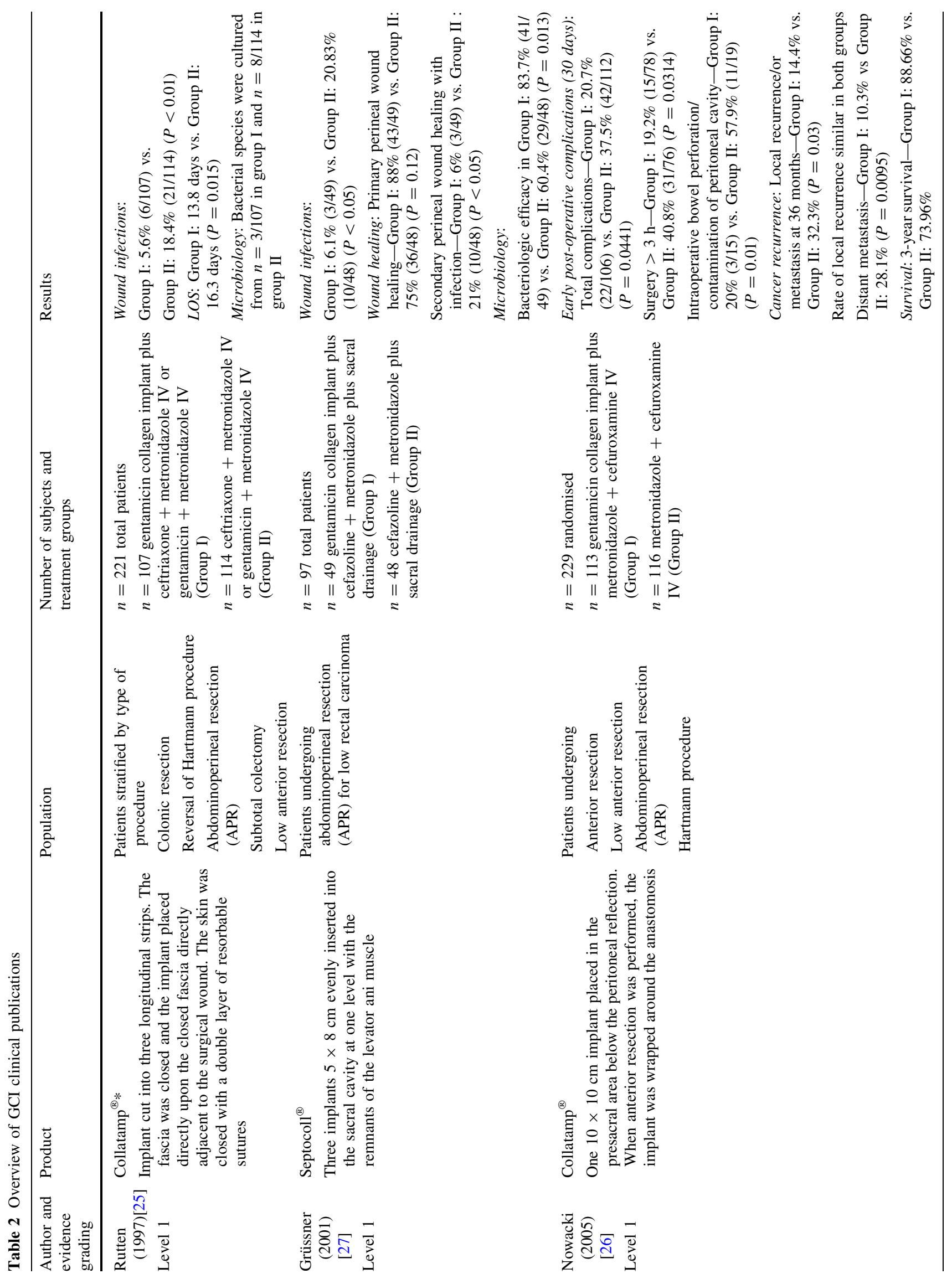




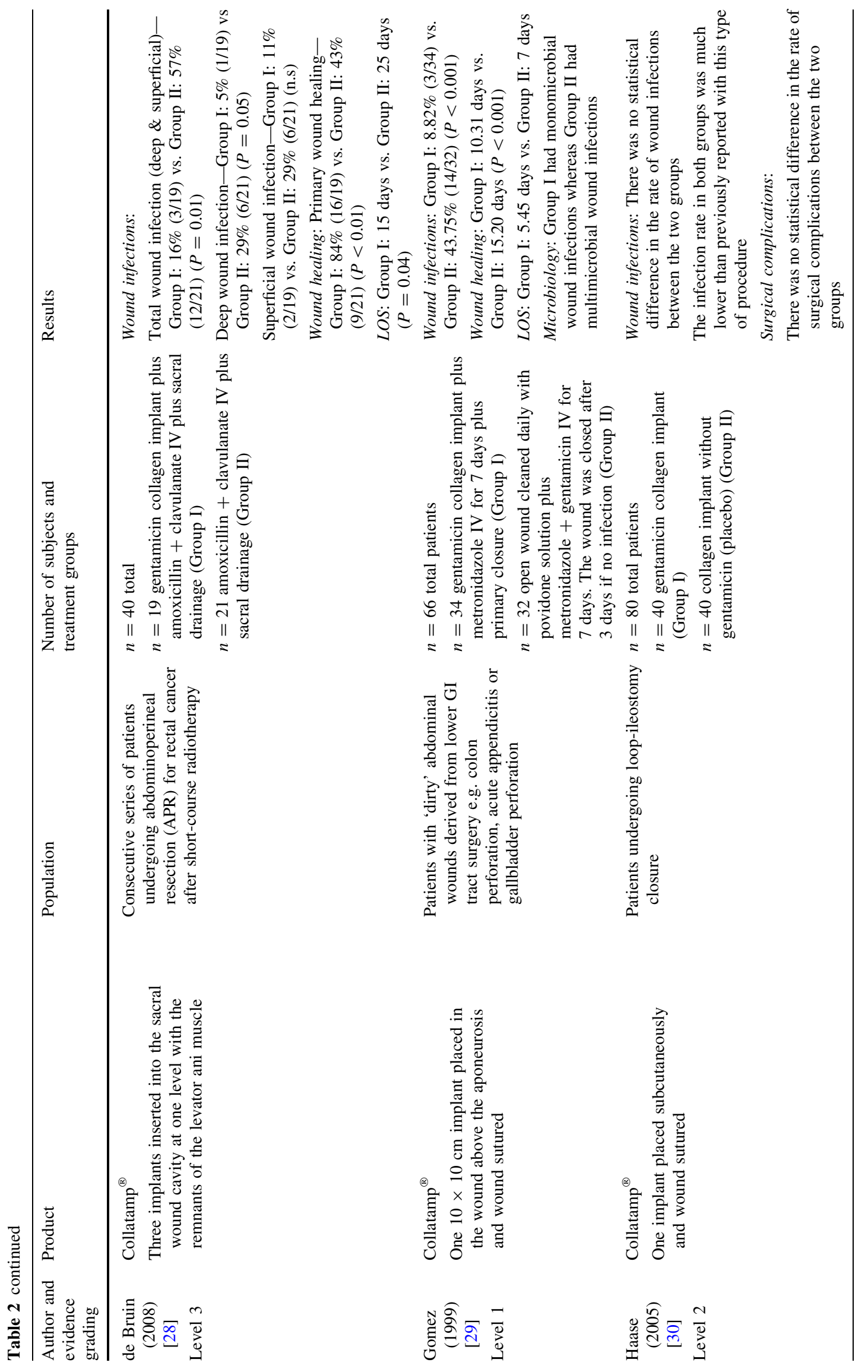




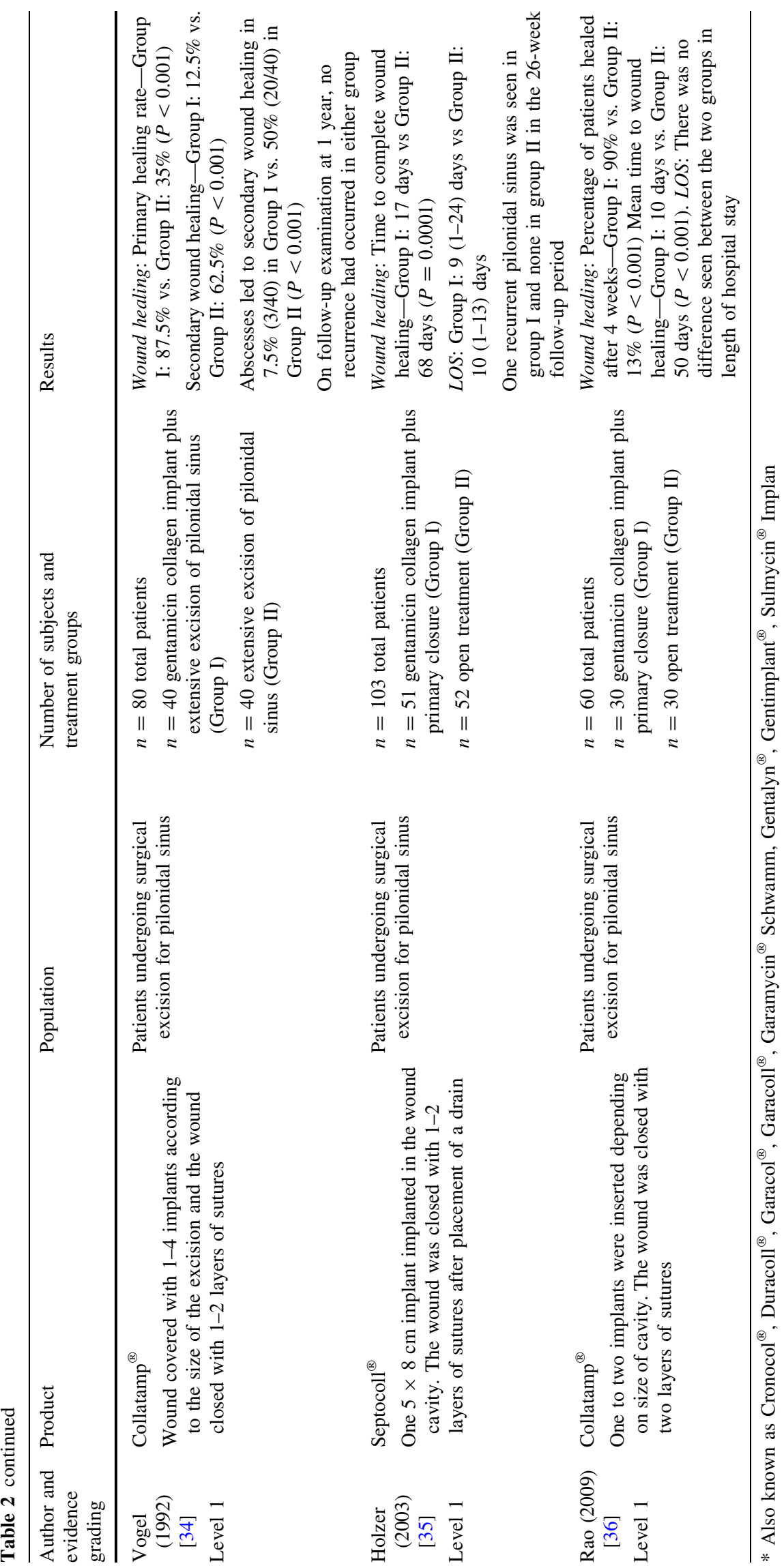


following loop-ileostomy closure [30] (Table 2). Eighty patients were randomised to the application of GCI or identical collagen implants without gentamicin. There was no statistical difference in the rate of wound infections or surgical complications between the two groups. The infection rate in both groups was much lower than previously reported with this type of procedure [31-33].

Pilonidal sinus excision

Three randomised studies have assessed the effectiveness of GCI in patients undergoing pilonidal sinus excision with primary wound closure [34-36]. Vogel and colleagues randomised 80 patients to the application of GCI with primary wound closure (Group I) or primary closure alone (Group II) [34]. The wound in both groups was closed with 1-2 layers of subcutaneous sutures (carefully avoiding any cavity formation) plus skin sutures. The primary healing rate was significantly higher in Group I with over twice the number of patients with wounds that healed post-operatively compared to Group II $(P<0.001)$. The patients in Group I also had a significantly lower secondary healing rate overall $(P<0.001)$. With this procedure, less than $10 \%$ of the patients had post-operative abscess formation in Group I, in contrast with over half of the patients in Group II $(P<0.001)$. At 1 -year follow-up, no recurrence had occurred in either group.

The second randomised study of pilonidal sinus excision randomised 103 patients to GCI plus primary closure (Group I) or open treatment (Group II), in order to compare surgical techniques for this indication [35]. In Group I, 14 (27\%) patients failed to achieve primary healing. Five of these required conversion to open treatment and 9 experienced spontaneous wound dehiscence. Thus overall 37 (73\%) patients experienced primary healing at 2 weeks. Wound breakdown was caused either by seroma formation or spontaneous dehiscence; however, complete healing of the failures by secondary intention occurred in almost all patients at 6 and 26 weeks.

Despite a failure rate of $27 \%$, the median time to complete wound healing was significantly shorter in Group I. Patients in Group II took almost four times as long to heal compared to patients in Group I $(P=0.0001)$. Despite the longer healing time in Group II, there was no significant difference between the two groups in mean length of hospital stay. One recurrent pilonidal sinus was seen in Group I and none in Group II in the 26-week follow-up period.

The third study of this indication for GCI use randomised 60 patients to GCI plus primary closure (Group I) or open treatment (Group II) and followed patients to assess recurrence rates at 5 years [36]. The excision with primary sutures utilised in Group I took a significantly longer time (mean: 23 min) than the open method used in Group II (mean: $14 \mathrm{~min})(P<0.0001)$.

Significantly more wounds in Group I had healed completely compared to Group II at 4 weeks $(P<0.001)$. Furthermore, the wounds in Group I healed in a significantly shorter time compared to those in Group II $(P<0.001)$. There was no difference seen between the two groups in length of hospital stay.

The closed wounds in Group I required fewer dressings (mean 2) when compared to open wounds in Group II (mean 25) $(P<0.001)$. The patients in Group I also experienced significantly less pain on post-operative days 2,4 and 7 (as reflected by their linear analogue pain scores) compared to the patients in Group II $(P<0.02)$. This resulted in less oral analgesic use in Group I. However, the difference between the two groups did not reach statistical significance at any time point.

Despite the lack of difference seen in length of hospital stay between the two groups, the overall cost per patient (calculated as hospital stay, cost of materials e.g. dressings and district nursing costs) was significantly lower for patients in Group I (mean of £149) compared to those in the open Group II (mean of £660) $(P<0.001)$. Recurrence rates were similar at 5-year follow-up.

\section{Discussion}

SSI is a common but potentially avoidable complication following GI surgery [37]. SSIs have significant impact on patient morbidity, mortality, QOL and resource utilisation and therefore, there is a need for more effective prevention in high-risk patients and procedures. There is a lack of evidence that prolonged prophylactic antibiotics reduce SSI rates and this approach may lead to antibiotic resistance. However, as early as 1997, Rutten and colleagues demonstrated the efficacy of locally applied gentamicin (with systemic antibiotics) in lowering the risk of antibiotic resistance.

This review has demonstrated that GCI can reduce the wound infection rate in high-risk GI surgery and improve wound healing after pilonidal sinus excision. GCI can also positively influence the post-operative course of patients undergoing particularly risky procedures e.g. APR combined with neoadjuvant radiotherapy. De Bruin and colleagues demonstrated that GCI reduced the wound infection rate by over $70 \%$ and the length of hospital stay by $40 \%$ in a series of patients who underwent pre-operative radiotherapy followed by APR [28].

Of the nine studies reviewed, one did not show any significant benefit of local application of GCI compared to systemic antibiotics alone. Haase et al. showed no additional benefit of GCI following loop-ileostomy closure. However, 
the study did have an extremely low SSI rate in the control group (10\%) in contrast to that found in the literature (up to $41 \%$ of patients) $[32-34,38,39]$. The lack of effect seen with GCI may therefore be related to the use of a collagen-only implant in the 'control' group which, as discussed before, may not be a true 'control' as collagen on its own has also been shown to positively impact wound healing.

Few adverse side effects were reported in these studies [26-31, 35, 36]. However, in our experience, the dissolution of the GCI may result in an increase in wound secretion but this does not seem to have further consequences for wound healing. Gentamicin is an aminoglycoside antibiotic that has the potential to induce severe nephrotoxicity and acute renal failure when used at high concentrations [40]. However, systemic concentrations of gentamicin with implantation of 1-5 GCI remain well below the established toxicity thresholds of $10-12 \mu \mathrm{g} / \mathrm{ml}$ for peak values and eventually fall below $2 \mu \mathrm{g} / \mathrm{ml}$ by $24 \mathrm{~h}$ [22]. In a multicentre trial of GCI in sternal wound infections, no difference in post-operative renal function was noted [41]. The effects of GCI in patients with chronic renal failure are still unknown and caution is warranted [42].

This review on the efficacy of GCI has brought up several topics which warrant further investigation. Binnebösel and colleagues have discovered that intra-abdominal applied gentamicin can enhance the healing of anastomosis and increase the collagen type I/type III in a rat model [43]. This may have positive implications for patients undergoing pilonidal sinus excision as the same group showed a decrease of extracellular matrix (ECM) and collagen in patients who with delayed wound healing [44]. Junge et al. also demonstrated that the quality of collagen formation was significantly increased when gentamicin was used to supplement a polyvinylidenflouride mesh material used in order to improve scar quality and mesh integration in hernia repair [45, 46]. Although this and other data suggest a positive effect of local gentamicin on collagen content and metabolism, there is conflicting data regarding this subject. Vaneerdeweg et al. studied the influence of GCI on the healing of colonic anastomoses in a rat model of peritonitis [47]. The rats where divided into four groups: control; collagen implant, intravenous (IV) gentamicin and GCI. No significant difference in bursting pressure was seen between the control, IV gentamicin and GCI groups. Almost all rats in the collagen group had anastomotic leakage, suggesting that collagen alone may be a focus for infection and anastomotic breakdown. The Vaneerdeweg data conflicts with that of Haase et al. who demonstrated that collagen had a beneficial effect in their 'collagen alone' group compared to the wound infection rate in the literature following loop-ileostomy closure. Therefore, further work is required to elicit the mechanism involved and the implications for GCI use.
Additional study is also required to evaluate the health economic benefit of GCI. The clinical data reviewed here has demonstrated a positive effect on the length of hospital stay with GCI after rectal tumour resection. In our recently published study, the difference in the length of hospital stay between the two groups was 10 days and this may indicate a potentially large saving in hospital costs per patient treated prophylactically with GCI following APR. In addition, overall costs were shown to be significantly reduced with GCI following pilonidal sinus excision in a small series of patients.

Utilising data from Rutten et al., and length of stay information from a UK-based survey on the adverse effects of SSI [48] the cost-benefit of GCI can be estimated. Based on 100 patients undergoing bowel surgery, the total cost saving due to reduction in length of hospital stay with GCI would be approximately $£ 16,000$. However, there has been no study to date which has utilised health economic endpoints in order to prospectively analyse the potential direct and indirect costs savings with GCI in high-risk GI surgical procedures.

\section{Conclusions}

Prophylactic use of gentamicin-containing collagen implants seems to be safe and simple to use, but does it work? This review demonstrates that GCI can have a positive effect on wound infection rates in high-risk GI surgery and can also improve wound healing after pilonidal sinus excision.

No serious side effects were noted in this review suggesting the prophylactic use of GCI in GI surgery is safe.

Due to the different patient populations, types of study and inclusion/exclusion criteria, a meta-analysis would be challenging to perform. Therefore, randomised trials with welldescribed patient categories, end points and follow-up could help to confirm the positive effect of GCI on wound healing, and at the same time evaluate their health economic benefit and investigate their potential effect on collagen metabolism.

Conflict of interest There is no financial relationship with the organisation that sponsored the research. We had full control of all primary data, and we agree to allow the journal to review our data if requested.

Open Access This article is distributed under the terms of the Creative Commons Attribution Noncommercial License which permits any noncommercial use, distribution, and reproduction in any medium, provided the original author(s) and source are credited.

\section{References}

1. Scottish Intercollegiate Guidelines Network (2008) Antibiotic prophylaxis in surgery. SIGN 104. July 2008 
2. Culver DH, Horan TC, Gaynes RP et al (1991) Surgical wound infection rates by wound class, operative procedure and patient risk index. National Nosocomial Infections Surveillance System. An J Med 91:152S-157S

3. ASA Physical Status Classification System. Available at http://www.asahq.org/clinical/physicalstatus.htm

4. Smith RL, Bohl JK, McElearney ST et al (2004) Wound infection after elective colorectal resection. Ann Surg 239:599-607

5. Health Protection Agency (2007) Surveillance of surgical site infection in England. CDR Weekly October 1997-September 2005

6. Suetens C, Wilson J, Ramboer I et al (1997) European surveillance of surgical site infections HELICS-SSI 2004-2005. European Scientific Conference on Applied Infectious Disease Epidemiology, Stockholm

7. Kapiteijn E, Marijnen CA, Nagtegaal ID et al (2001) Preoperative radiotherapy combined with total mesorectal excision for resectable rectal cancer. N Engl J Med 345:638-646

8. Bullard KM, Trudel JL, Baxter NN, Rothenberger DA (2005) Primary perineal wound closure after preoperative radiotherapy and abdominoperineal resection has a high incidence of wound failure. Dis Colon Rectum 48:438-443

9. Song F, Glenny AM (1998) Antimicrobial prophylaxis in colorectal surgery: a systematic review of randomized controlled trials. Br J Surg 85:1232-1241

10. Morita S, Nishisho I, Nomura T et al (2005) The significance of the intraoperative repeated dosing of antimicrobials for preventing surgical wound infection in colorectal surgery. Surg Today 35:732-738

11. Fujita S, Saito N, Yamada T et al (2007) Randomized, multicenter trial of antibiotic prophylaxis in elective colorectal surgery: single dose vs 3 doses of a second generation cephalosporin without metronidazole and oral antibiotics. Arch Surg 142:657661

12. Suehiro T, Hirashita T, Araki S et al (2008) Prolonged antibiotic prophylaxis longer than 24 hours does not decrease surgical site infection after elective gastric and colorectal surgery. Hepatogastroenterology 55:1636-1639

13. Stemberger A, Grimm H, Bader F et al (1997) Local treatment of bone and soft tissue infections with the collagen-gentamicin sponge. Eur J Surg S578:17-26

14. Bendavid R (1997) The Shouldice technique: a canon in hernia repair. Can J Surg 40:199-205

15. Chvapil M, Kronenthal RL, van Winkle W Jr (1973) Medical and surgical applications of collagen. Int Rev Connect Tissue Res 6:1-55

16. Chvapil M (1977) Collagen sponge: Theory and practice of medical applications. J Biomed Mater Res 11:721-741

17. Anselme K, Bacques C, Charriere G et al (1990) Tissue reaction to subcutaneous implantation of collagen sponge. A histological, ultrastructural, and immunological study. J Biomed Mater Res 24:689-703

18. Kinel FA, Ciaccio LA, Henderson SB (1984) Sustained release preparations, XVI:Collagen as a drug carrier. Arch Pharm 317:657-661

19. Prior DV (2004) Localised drug delivery via collagen-based biodegradable matrices. The Drug Delivery Companies Report $39-42$

20. Waldvogel FA (1984) Future perspectives of aminoglycoside therapy. J Antimicrob Chemother 13:75-78

21. Moore RD, Lietman PS, Smith CR (1987) Clinical response to aminoglycoside therapy: importance of the ratio of peak concentration to minimal inhibitory concentration. J Infect Dis 155:93-99

22. Ruszczak Z, Friess W (2003) Collagen as a carrier for on-site delivery of antibacterial drugs. Adv Drug Deliv Rev 55:16791698
23. Blaser J, Stone BB, Groner MC, Zinner SH (1987) Comparative study with enoxacin and netilmicin in a pharmacodynamic model to determine importance of ratio of antibiotic peak concentration to MIC for bactericidal activity and emergence of resistance. Antimicrob Agents Chemother 31:1054-1060

24. Carruthers SG, Larochelle P, Haynes RB et al (1993) Report of the Canadian hypertension society consensus conference. I. Introduction. CMAJ 149:289-292

25. Rutten HJT, Nijhuis PHA (1997) Prevention of wound infection in elective colorectal surgery by local application of gentamicincontaining collagen sponge. Eur J Surg S578:31-35

26. Nowacki MP, Rutkowski A, Olędzki J, Chwaliński M (2005) Prospective, randomized trail examining the role of gentamycincontaining collagen sponge in the reduction of postoperative morbidity in rectal cancer patients: early results and surprising outcome at 3-year follow-up. Int J Colorectal Dis 20:114-120

27. Gruessner U, Clemens M, Pahlplatz PV et al (2001) Improvement of perineal wound healing by local administration of gentamicinimpregnated collagen fleeces after abdominoperineal excision of rectal cancer. Am J Surg 182:502-509

28. De Bruin AFJ, Gosselink MP, Wijffels NAT, Coene PP, van der Harst E (2008) Local gentamicin reduces perineal wound infection after radiotherapy and abdominoperineal resection. Tech Coloproctol 12:303-307

29. Gomez GGV, Guerrero TS, Lluck MC, Delgado FJ (1999) Effectiveness of collagen-gentamicin implant for treatment of 'dirty' abdominal wounds. World J Surg 23:123-127

30. Haase O, Raue W, Böhm B, Neuss H, Scharfenberg M, Schwenk W (2005) Subcutaneous gentamycin implant to reduce wound infections after loop-ileostomy closure: a randomized, doubleblind, placebo-controlled trial. Dis Colon Rectum 48:2025-2031

31. Riesener KP, Lehnen W, Hofer M et al (1997) ) Morbidity of ileostomy and colostomy closure: impact of surgical technique and perioperative treatment. World J Surg 21:103-108

32. Kohler A, Athanasiadis S, Nafe M (1994) Postoperative results of colostomy and ileostomy closure. A retrospective analysis of three different closure techniques in 182 patients. Chirurg 65:529-532

33. Hackam DJ, Rotstein OD (1995) Stoma closure and wound infection-an evaluation of risk-factors. Can J Surg 38:144-148

34. Vogel P, Lenz J (1992) Treatment of pilonidal sinus with excision and primary suture using a local, resorbable antibiotic carrier. Results of a prospective randomized trial. Chirurg 63:748753

35. Holzer B, Grüßner B, Brückner M et al (2003) Efficacy and tolerance of a new gentamicin collagen fleece (Septocoll) after surgical treatment of pilonidal sinus. Colorectal Dis 5:222-227

36. Rao MM, Zawislak W, Kennedy R, Gilliland R (2009) A prospective randomized study comparing two treatment modalities for chronic pilonidal sinus with a 5-year follow-up. Int J Colorectal Dis [E-pub ahead of print]

37. Deysine M (2006) Infection control in a hernia clinic: 24 year results of aseptic and antiseptic measure implementation in 4, 620 'clean cases'. Hernia 10:25-29

38. Hasegawa H, Radley S, Morton DG, Keighley MR (2000) Stapled versus sutured closure of loop ileostomy-a randomized controlled trial. Ann Surg 231:202-204

39. Edwards DP, Leppington-Clarke A, Sexton R et al (2001) Stomarelated complications are more frequent after transverse colostomy than loop ileostomy: a prospective randomized clinical trial. Br J Surg 88:360-363

40. British Medical Association \& Royal Pharmaceutical Society of Great Britain. British National Formulary, 57th edn, March 2009

41. Friberg O, Svedjeholm R, Kallman J, Söderquist B (2007) Incidence, microbiological findings, and clinical presentation of sternal wound infections after cardiac surgery with and without 
local gentamicin prophylaxis. Eur J Clin Microbiol Infect Dis 26:91-97

42. Collatamp ${ }^{\circledR}$ Summary of Product Characteristics

43. Binnebösel M, Junge K, Kaemmer DA et al (2009) Intraperitoneally applied gentamicin increases collagen content and mechanical stability of colon anastomosis in rats. Int $\mathrm{J}$ Colorectal Dis $24: 433-440$

44. Binnebösel M, Junge K, Schwab R et al (2009) Delayed wound healing in sacrococcygeal pilonidal sinus coincides with an altered collagen composition. World J Surg 33:130-136

45. Junge K, Rosch R, Klinge U et al (2005) Gentamicin supplementation of the polyvinylidenflouride mesh materials for infection prophylaxis. Biomaterials 26:787-793
46. Junge $\mathrm{K}$, Klinge U, Rosch R et al (2007) Improved collagen type I/ III ratio at the interface of gentamicin-supplemented polyvinylidenflouride mesh materials. Langenbecks Arch Surg 392:465471

47. Vaneerdeweg W, Hendriks JM, Lauwers PR, Ieven M, Eyskens EJ (2000) Effect of gentamicin-containing sponges on the healing of colonic anastamoses in a rat model of peritonitis. Eur J Surg 166:959-962

48. Coello R, Charlett A, Wilson J et al (2005) Adverse impact of surgical site infections in English hospitals. J Hosp Infect 60:93-103 